\title{
Transporte público, bienestar y desigualdad: cobertura y capacidad de pago en la ciudad de Montevideo
}

\author{
Diego Hernández
}

\section{Resumen}

\begin{abstract}
El transporte público es clave para garantizar la capacidad de las personas para acceder a las oportunidades que ofrece la ciudad. A diferencia de otros medios de transporte, como el automóvil, este es el que menos peso monetario recarga sobre los usuarios, lo que lo convierte en el medio que más desmercantiliza la movilidad.

En este trabajo se analizan los niveles de cobertura territorial del transporte público, así como la capacidad de pago por el servicio en la ciudad de Montevideo. Además, se buscan pautas de estratificación entre grupos socioeconómicos diversos.

Los indicadores presentados dan cuenta de niveles muy altos de cobertura básica de la red, lo que coincide con un patrón de crecimiento asociado al seguimiento de la demanda espacial y horaria. Esta cobertura presenta varios matices de acceso estratificado, en particular al incorporar la dimensión de la capacidad de pago. La evidencia indica que el gasto de los hogares registra una distribución regresiva en materia de acceso.
\end{abstract}

Palabras clave

Transporte público, política de transporte, bienestar social, igualdad, gastos familiares, ciudades, Uruguay

Clasificación JEL

R40, R48, Z13, Z18

Autor

Diego Hernández es Profesor Asistente del Departamento de Ciencias Sociales y Políticas de la Universidad Católica del Uruguay. dhernandez@ucu.edu.uy 


\section{Introducción}

La movilidad urbana facilita el acceso a otros bienes y oportunidades, por lo que tiene un impacto relevante en la calidad de vida de las personas. De hecho, juega un destacado papel en la producción y reproducción de las estructuras sociales, que, al mismo tiempo, repercuten en las características de la movilidad cotidiana (Cass, Shove y Urry, 2005; Kaufmann, 2002; Manderscheid, 2009; Ohnmacht, Maksim y Bergman, 2009). La movilidad no es un tema de libre elección personal y puede presentar limitaciones estructurales muy fuertes (Massot y Orfeuil, 2005; Wenglenski y Orfeuil, 2006). Sería ingenuo pensar que la movilidad llega a todos los estratos sociales por igual; no se debe confundir el hecho de que la mayor velocidad de transporte y movimiento sea un imperativo de las sociedades modernas con que, efectivamente, toda la población acceda a ella (Kaufmann, 2002; Urry, 2007). En pocas palabras, la movilidad no se distribuye de forma equitativa: hay personas que tienen más posibilidades de movilizarse que otras, hay quienes pueden movilizarse mucho más rápido y en más direcciones.

La accesibilidad a bienes, servicios y oportunidades es clave para el bienestar de las personas y, por ese motivo, convierte al transporte público en una política social similar a muchas otras de mayor "tradición", como la salud o la educación. Este es el dispositivo desmercantilizador más relevante de la accesibilidad, lo que significa que una persona que tiene que desplazarse en la ciudad encuentra en el transporte público la forma más económica de hacerlo. Más allá de que implica gastos de bolsillo (pagar una tarifa), el transporte público es el medio que menos capacidad de pago le demanda al individuo. Esa menor demanda supone que, en cierta medida, sea más independiente del desempeño de la persona en el mercado laboral. La desmercantilización parte de su concepción como un derecho social y no como un producto con valor monetario que solo depende de los vaivenes del mercado.

Por tanto, como otras políticas sociales, el transporte público debe analizarse desde tres variables básicas: i) el nivel de cobertura; ii) la capacidad de pago por el servicio, y iii) la existencia de mecanismos específicos de desmercantilización financiera. En este artículo se analizan las tres variables para el caso de la ciudad de Montevideo desde una perspectiva de bienestar y equidad en la provisión de un bien social.

Este artículo constituye un aporte al estudio de la movilidad urbana en la región desde la perspectiva de estructuras de bienestar y políticas sociales. Asimismo, contribuye al análisis de las políticas sociales en el continente, en tanto analiza una política sectorial que siempre se menciona como central, aunque rara vez se estudia de forma sistemática en busca de lógicas propias de provisión y estratificación.

Para cumplir con sus objetivos, el artículo se estructura como se establece a continuación. En la sección II se presenta el marco conceptual que sirve de guía, con especial énfasis en el papel de la accesibilidad en el bienestar de las personas y la función desmercantilizadora del transporte público (para ello se recurre al esquema de activos y estructuras de oportunidades). En la sección III se realiza un breve análisis del caso de la ciudad de Montevideo y se describe la metodología utilizada para la construcción de los indicadores analizados. En la sección IV se presentan los resultados en materia de cobertura y capacidad de pago por estrato socioeconómico. Esta evidencia se complementa con la revisión de los mecanismos desmercantilizadores identificados para el sistema de transporte montevideano. El artículo se cierra con una discusión de la evidencia empírica en la sección V. 


\section{Marco conceptual: accesibilidad, bienestar y transporte público}

\section{El concepto de accesibilidad}

Para Miralles y Cebollada, la accesibilidad: “... hace referencia a la facilidad con que cada persona puede superar la distancia que separa dos lugares y de esta forma ejercer su derecho como ciudadano..." (Miralles-Guasch y Cebollada, 2003, pág. 14)1. Vale destacar que un individuo puede tener muy poca capacidad de movilizarse a grandes distancias en el espacio y, sin embargo, tener muy buena accesibilidad por cercanía. Y a la inversa, puede tener facilidad de movimiento hacia muchos puntos de la ciudad, pero no hacia el que necesita llegar, por lo que, a pesar del alto nivel de movilidad, su accesibilidad sigue siendo baja. También puede registrar muchos movimientos — ser muy "móvil" pero, debido a que tiene que transitar distancias largas, su accesibilidad es, en realidad, deficitaria. En tal sentido, la localización de las actividades juega un papel relevante. Esto supone que, en teoría, la superación de las distancias puede darse desde el individuo desplazándose a las actividades o desde las actividades "desplazándose" hacia el individuo (localización cercana). Los problemas de accesibilidad al trabajo, por ejemplo, podrían resolverse de dos formas: con un adecuado sistema de transporte que lleve a las personas a las zonas con alta concentración de oportunidades laborales o a través del emplazamiento de oportunidades cercanas al lugar de residencia.

A partir de los estudios sobre movilidad, accesibilidad y pobreza se ha identificado un conjunto de variables que facilitan u obstaculizan el acceso (Cass, Shove y Urry, 2005; Church, Frost y Sullivan, 2000; Flamm, Jemelin y Kaufmann, 2008; Titheridge, 2006; Urry, 2007). Al concentrarse en el transporte público, estas se podrían sintetizar en las siguientes dimensiones²:

i) Oportunidades de transporte: cuál es la red vial y su extensión, así como la extensión de la red de transporte público, los tiempos de desplazamiento, las características de la oferta pública colectiva (horarios, regularidad, extensión temporal), la calidad en términos de comodidad y seguridad tanto del material rodante como estático, y la información que se pone a disposición de los individuos.

ii) Configuración institucional: cuál es la participación estatal en la configuración de las oportunidades de transporte y la definición de quiénes pagan y quiénes reciben beneficios en relación con los costos de los desplazamientos. También se pregunta por la prioridad que se otorga al transporte privado respecto del transporte público (o viceversa), los costos de circulación (por ejemplo, si existen autopistas o vías urbanas de pago, peajes y demás), la estructura tarifaria del transporte público y la existencia de mecanismos de regulación y subsidios.

iii) Rasgos de los individuos: se define cuánto podrán aprovechar los individuos las oportunidades de transporte y el lugar que ocupan en relación con la configuración institucional. En tal sentido, deberán considerarse los ingresos de las personas, el tiempo disponible y cómo pueden organizarlo (en relación con sus actividades), y las habilidades y destrezas para dominar el sistema y su funcionamiento (por ejemplo, saber manejar o poder entender cómo funciona el transporte público) y poder acceder físicamente al transporte.

\footnotetext{
1 Otro concepto que, si bien difiere terminológicamente, tiene mucho que ver con la accesibilidad es el de motilidad aportado por Kaufmann, Manfred y Joye, a la que definen como "la capacidad de ser móvil en el espacio social y geográfico o como la manera en que las entidades, de acuerdo a sus circunstancias, acceden y se apropian de la capacidad de movilidad socioespacial" (Kaufmann, Manfred y Joye, 2004, pág. 750).

2 Adaptado de Hernández (2012).
} 
iv) Forma urbana: se relaciona con dinámicas socioterritoriales como la localización de los hogares y de las oportunidades en la ciudad.

Estas dimensiones cubren varios aspectos estructurales y diversas características individuales en cuya combinación se define la capacidad de las personas para superar las distancias geográficas. Por ejemplo, una persona puede vivir en una zona con excelente provisión de transporte público, pero si no puede pagar la tarifa, su accesibilidad será limitada o deficitaria.

A fin de captar esta interacción entre aspectos individuales y estructurales se puede recurrir al paradigma de activos y estructuras de oportunidades (Hernández, 2012). El análisis de este enfoque se centra en el concepto de vulnerabilidad como herramienta que permite trascender la visión dicotómica pobre-no pobre a la hora de analizar el bienestar de los hogares y las personas.

\section{Activos y estructuras de oportunidades de movilidad}

Como se indicó, el enfoque de vulnerabilidad-activos surge de la necesidad de conocer más sobre la heterogeneidad de la pobreza y la lógica de actuación de los hogares de menores ingresos. Propone que hay que trascender la pregunta de si un hogar es pobre o no. Desde este enfoque, lo relevante es cuestionarse acerca de la capacidad del hogar para enfrentar situaciones de riesgo que comprometan niveles básicos de bienestar. En otras palabras, debe conocerse la vulnerabilidad de estos hogares. Moser (1998) define a la vulnerabilidad como el riesgo del bienestar de individuos y hogares frente a un ambiente cambiante, así como su capacidad de respuesta y recuperación ante cambios desfavorables. Esta autora afirma que, conociendo el portafolio de activos y sus estrategias de utilización, es posible facilitar la intervención social para que los hogares pobres puedan utilizar sus activos de forma "productiva".

Kaztman (1999) plantea que es necesario prestar más atención a las estructuras de oportunidades de una sociedad, ya que el grado de vulnerabilidad de un hogar estaría dado por el desfasaje entre estas y su portafolio de activos. Siguiendo a Kaztman, "el concepto de activos no alcanza una significación unívoca si no está referido a las estructuras de oportunidades que se generan desde el lado del mercado, la sociedad y el Estado. En otras palabras, se sostiene que el portafolio y la movilización de activos de los hogares vulnerables, punto central en la formulación moseriana, solo puede examinarse a la luz de las lógicas generales de producción y reproducción de activos, que no pueden ser reducidas a la lógica de las familias y sus estrategias” (Kaztman, 1999, pág. 34).

En líneas generales, este esquema propone la existencia de estructuras de oportunidades que se definen como probabilidades de acceso a bienes, a servicios o al desempeño de actividades. Estas oportunidades inciden sobre el bienestar de los hogares porque permiten o facilitan a sus integrantes el uso de sus propios recursos (Kaztman, 1999, pág. 21). Las oportunidades pueden provenir de las tres grandes instituciones del orden social: el mercado, el Estado y la sociedad (véase el diagrama 1).

El aprovechamiento de estas estructuras de oportunidades dependerá de los recursos y activos de los hogares. Los recursos son todos los bienes que controla un hogar, ya sean tangibles o intangibles, mientras que la idea de activo (también se podría usar el término "capital") se refiere al subconjunto de esos recursos cuya movilización permite el aprovechamiento de las estructuras de oportunidades existentes en un momento, ya sea para elevar el nivel de bienestar o para mantenerlo ante situaciones que lo amenazan (Kaztman, 1999, págs. 31-32). A partir de este enfoque se podría identificar una tensión entre los recursos bajo el control de los hogares y las estructuras de oportunidades que responden a limitaciones del medio. Como afirman los autores de esta línea, considerar los activos sin tener en cuenta las estructuras de oportunidades desdibuja su significado, por lo que hay que trascender el análisis de las estrategias de movilización de activos, complementarlo con el estudio del acceso a las estructuras de oportunidades y revisar si este sigue algún tipo de pauta 
de estratificación. Este contraste, ajuste o adaptación es el que define la vulnerabilidad de los hogares (Kaztman, 1999 y 2009; Kaztman y Filgueira, 2006).

\section{Diagrama 1}

Esquema de activos y estructuras de oportunidades de accesibilidad

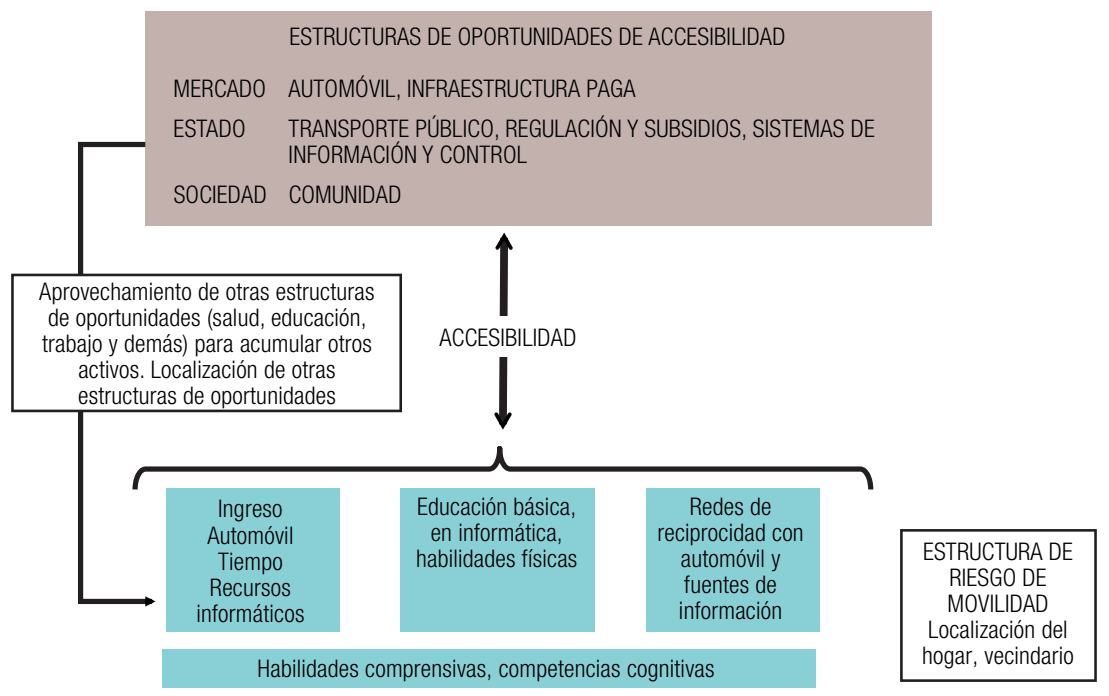

Fuente: D. Hernández, "Activos y estructuras de oportunidades de movilidad. Una propuesta analítica para el estudio de la accesibilidad por transporte público, el bienestar y la equidad", EURE. Revista Latinoamericana de Estudios Urbano Regionales, vol. 38, № 115, Santiago, Pontificia Universidad Católica de Chile, 2012.

Los recursos que los hogares pueden movilizar (y convertir en capital) responden a la tradicional clasificación en capital físico (ingresos), capital humano (nivel educativo) y capital social (participación en redes de reciprocidad).

Al repasar la lógica de este esquema, se verifica que la accesibilidad se ubica en el punto de encuentro entre las estructuras de oportunidades y los recursos con que cuentan los hogares para aprovecharlas. La vulnerabilidad o el desajuste entre ambas variables podrán estar dados por una o la otra. Así, por ejemplo, podría derivarse una estructura típica de alto riesgo de movilidad constituida por aquellos hogares pobres sin opciones motorizadas privadas. De existir una oferta de transporte público, los hogares que no cuenten con ingresos suficientes para pagar la tarifa, difícilmente podrán sacar provecho de ella. En este caso, esta vulnerabilidad podría resolverse desde el transporte público a través de la implementación de mecanismos financieros compensatorios o bien modificando los recursos con que cuentan los hogares (aumentando sus ingresos). Por supuesto que queda descartado de plano que estos hogares puedan aprovechar oportunidades provenientes de la esfera del mercado, ya que, si no cuentan con capacidad de pago para el transporte público, difícilmente podrían transitar, por ejemplo, por autopistas pagas.

Uno de los elementos más relevantes para este artículo es que las oportunidades provenientes del transporte público se colocan en el orden estatal por el papel protagónico del Estado - que podrá asumir o no- en la regulación y determinación del diseño del bien a proveer, incluida, por supuesto, la dimensión tarifaria. Su papel central en la discusión, no obstante, responde principalmente a que es el mecanismo motorizado con mayor potencial de desmercantilización de la movilidad y es allí donde debe centrarse la discusión. Esta noción de desmercantilización (Esping-Andersen, 1990) está vinculada a la capacidad del individuo de acceder al bienestar con independencia de su desempeño en el mercado. 
¿Cómo se manifiesta la desmercantilización? En un escenario de máxima desmercantilización, la capacidad de pago del individuo es prácticamente indiferente respecto de la capacidad de utilizar el sistema. En un escenario de mínima desmercantilización, su capacidad para desplazarse de un punto a otro de la ciudad dependerá, de forma casi exclusiva, de su capacidad de pago, que a su vez está vinculada a su desempeño en el mercado laboral o a su capital físico heredado. En un contexto de alta mercantilización, entonces, la capacidad de movilidad motorizada de un individuo desempleado y carente de ahorros $u$ otros ingresos es prácticamente nula. De hecho, en este extremo, donde no existen elementos regulatorios de la tarifa, se podría incluso argumentar que no existen oportunidades de movilidad desde el Estado más allá de la infraestructura vial básica. En el escenario opuesto, la capacidad de pago no sería un dato relevante en tanto el uso no demandaría gastos de bolsillo. En el escenario de máxima desmercantilización, el financiamiento de la provisión del bien provendría de rentas generales, por lo que elementos como el régimen impositivo, las estructuras de subsidio y la focalización se vuelven relevantes. Claro está que estos extremos no son reales y que la discusión relevante está en la definición de las situaciones intermedias (véase el diagrama 2).

\section{Diagrama 2}

Representación gráfica de los extremos de desmercantilización a partir del transporte público

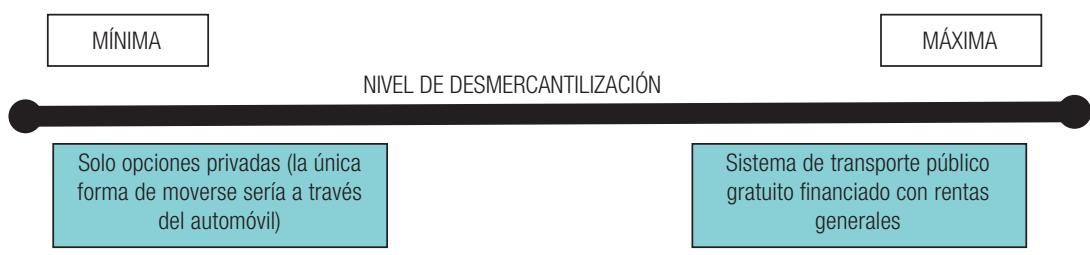

Fuente: Elaboración propia.

Además del aspecto del financiamiento, es necesario tener en cuenta quién es el que efectivamente se beneficia de lo "producido" por el transporte público y con qué calidad. De nada serviría un sistema gratuito y de excelente calidad, pero con una cobertura territorial mínima. De la misma forma, tampoco sería efectivo un sistema muy amplio en cobertura y de muy bajo costo - o incluso gratuito - , pero cuya calidad (velocidad, regularidad, seguridad y demás) fuera pésima.

En otras palabras, como ocurre en otras políticas sociales, las preguntas que hay que responder en el caso del transporte público son tres: quién paga (cuánto y cómo se financia), quién accede al bien y cuál es la calidad de dicho bien. En este artículo se responden dos preguntas centrales a este respecto: quiénes están cubiertos y cuánto pagan por el servicio. Si bien no agotan las preguntas pertinentes sobre desmercantilización, estas respuestas echan luz sobre su dinámica de desigualdad en el acceso a este bien, así como de eventuales mecanismos que intenten revertirla.

\section{Presentación del caso y aspectos metodológicos}

Montevideo es la capital del Uruguay y, si bien es el departamento más pequeño, sus 1,3 millones de habitantes representan algo menos de la mitad de la población total del país. Se trata de una ciudad costera que es el centro histórico y distrito de negocios del país. Se podría afirmar que tiene una estructura urbana monocéntrica. La red de transporte público absorbe algo más de 1 millón de viajes diarios y cuenta con unos 1.500 autobuses, 4.792 paradas y 3 estaciones de intercambio con infraestructura completa. Se organiza en torno a 136 recorridos que acumulan unas 1.066 variantes que incluyen variantes en ambos sentidos, variantes más cortas, aunque significativas, del recorrido original y, en escasas ocasiones, variantes en cuanto al recorrido propiamente dicho (Urse y Márquez, 
2009; Intendencia de Montevideo, 2010). La enorme mayoría de estos recorridos es de carácter regular (no local o diferencial) y tiene como origen o destino alguna zona céntrica ${ }^{3}$.

Los objetivos de esta investigación son descriptivos para las variables analizadas y sus pautas de estratificación socioeconómica. La evidencia empírica proviene de varias fuentes de información de carácter espacial y longitudinal. En el caso de la cobertura de transporte, se ejecutaron geoprocesamientos de información espacial proveniente de la Intendencia de Montevideo y el Instituto Nacional de Estadística. Concretamente, se trabajó con las estaciones de autobús (paradas) en relación con los centroides de cada una de las manzanas. De esta forma, a partir de áreas de influencia calculadas con la distancia euclidiana entre cada punto, se determinó la presencia o no de una parada respecto de ese centroide. Para el cálculo de la población cubierta se utilizó la metodología usada por Gutiérrez y García (2005), que consiste en calcular la proporción de la zona que está cubierta por el área de influencia y aplicarla sobre la cantidad de personas que viven en ella, asumiendo que estas se encuentran cubiertas por dicha área de influencia. Por ejemplo, si en una zona censal donde viven 100 personas, el buffer dibujado a partir de la parada cubre el 50\% del territorio, se asume que las personas cubiertas por esa área de influencia son 50. En este caso, si el área cubierta alcanzara el $90 \%$, se asumiría que la totalidad de las personas estarían cubiertas. Los datos sobre frecuencia teórica del servicio de autobuses provienen de la información publicada en la página web de la Intendencia de Montevideo.

Para los datos sobre capacidad de pago y gasto en transporte se utilizó la Encuesta Nacional de Gastos e Ingresos de los Hogares del Instituto Nacional de Estadística. Para la mayor parte de la información se trabajó con los gastos de transporte urbano, una categoría que excluye el transporte por esparcimiento y otros (por ejemplo, los viajes de placer al exterior).

El estrato socioeconómico surge de la división en quintiles de los ingresos de los hogares montevideanos. En el caso del cálculo espacial, los estratos responden a la proporción de hogares en los dos quintiles más pobres, que se calcula sobre el segmento censal (aproximadamente seis manzanas en la trama urbana) y se imputa a cada manzana que lo compone.

\section{Resultados}

\section{Cobertura de la red de transporte público}

La red presenta una densidad considerable en el conjunto de la ciudad y deja escasos intersticios de territorio poblado sin cobertura. En líneas generales, su desarrollo detrás de la demanda se podría catalogar como relativamente exitoso en cuanto a la extensión. La enorme mayoría de las zonas con mayor densidad de población cuentan con algún recorrido de transporte público que las atraviesa. Muchas de las zonas que a primera vista no aparecen atendidas pertenecen al llamado "Montevideo rural". Se trata de una red verdaderamente densa que, con mayor o menor cantidad de servicios, parece llegar a todas las áreas con potencial demanda en la ciudad. En tal sentido, se podría afirmar que Montevideo es una ciudad con una muy alta conectividad proveniente del transporte público.

Al observar las áreas de influencia construidas alrededor de las paradas del sistema, es notorio que el centro y la costa este parecen estar uniformemente pintados con el indicador de 300 metros de distancia de alguna parada, cuadro que solo se interrumpe de forma muy esporádica con pequeñas porciones de territorio donde la distancia hacia la parada más cercana es entre 300 metros y 500 metros (véase el mapa 1). En las restantes regiones del departamento la situación es

\footnotetext{
3 En particular, la zona del distrito de negocios conocido como Ciudad Vieja (o Aduana) o su "puerta de entrada", la Plaza Independencia.
} 
algo más heterogénea en tanto se comienzan a vislumbrar determinadas zonas a más de 500 metros de alguna parada e incluso más allá de los 750 metros. En la periferia, las áreas de influencia son aún bastante continuas, con excepción de la zona oeste. En la periferia suburbana, las áreas de influencia comienzan a "seguir" la lógica de los recorridos que penetran en ella de forma radial y es mucho menos advertible una lógica transversal.

Mapa 1

Montevideo: ubicación de las paradas y áreas de influencia (300, 500 y 750 metros)

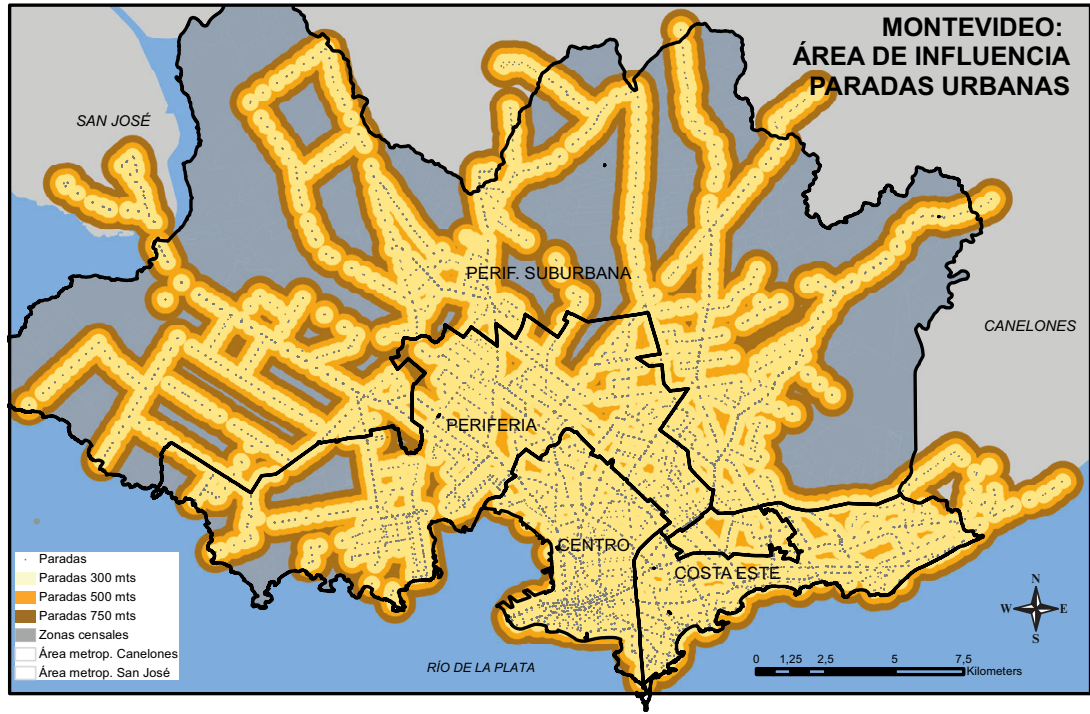

Fuente: Elaboración propia, sobre la base de información de la Intendencia de Montevideo y el Instituto Nacional de Estadística.

Como se observa en el cuadro 1, la cantidad de personas sin una estación de transporte a 750 metros o menos es marginal en términos proporcionales. Al detenerse en el porcentaje del territorio de la ciudad que se encuentra a esa distancia, es fácilmente deducible la muy baja densidad poblacional de las zonas con grandes obstáculos al acceso básico. Ello constituye una muestra de la efectividad del sistema en el seguimiento de la demanda, así como de su capacidad para evitar extenderse hacia zonas de escasa demanda potencial.

Cuadro 1

Montevideo: área y población, según umbrales de distancia a la red de transporte público (En porcentajes, personas y metros)

\begin{tabular}{lcccc}
\hline Distancia a la parada & $\begin{array}{c}\text { Área } \\
\text { (en porcentajes) }\end{array}$ & $\begin{array}{c}\text { Personas } \\
\text { (en porcentajes) }\end{array}$ & Cantidad de personas & Distancia media $^{\mathrm{a}}$ \\
\hline Más de 300 metros & 53,70 & 8,83 & 117045 & 604 \\
\hline Más de 500 metros & 38,72 & 2,17 & 28752 & 1006 \\
\hline Más de 750 metros & 26,48 & 0,70 & 9348 & 1322 \\
\hline
\end{tabular}

Fuente: Elaboración propia, sobre la base de información de la Intendencia de Montevideo y el Instituto Nacional de Estadística.

a Se trata de la distancia media de los centroides de las zonas censales que no son intersectadas por el área de influencia de la parada en cada uno de los umbrales (300 metros, 500 metros y 750 metros). Aquellas zonas que son intersectadas al menos parcialmente no se tienen en cuenta para el cálculo.

La capacidad para evitar la extensión hacia zonas de escasa demanda potencial se traduce en un elemento, a priori, positivo, ya que son muy escasos los territorios con necesidad de transporte a los que el sistema no haya llegado de una forma u otra. Al pasar a un nivel un poco más alto 
- aunque aún moderado - de obstáculos al acceso al transporte público (paradas a más de 500 metros), la cantidad de personas sin cobertura aumenta y llega a algo más del 2\% de la población montevideana. Una vez más, el área territorial en esta situación sugiere una muy baja densidad poblacional, no obstante lo cual, resulta claro que en este caso se trata de sectores con densidad poblacional sustancialmente mayores que los de la situación anterior. Vale recordar que en este estrato ya comienzan a registrarse casos en todas las regiones, con algo de mayor intensidad en la periferia y en la franja sur de la periferia suburbana.

En pocas palabras, al analizar el acceso básico a las estaciones de transporte público en Montevideo, el primer dato que debe tenerse presente es que prácticamente 9 de cada 10 personas residen en una zona censal que tiene una parada a 300 metros o menos. Esto habla de una muy alta conectividad del territorio en la ciudad y su gente, aunque también da cuenta de algunas zonas que, debido a su localización, presentan determinados obstáculos leves para alrededor del 9\% de la población (que está a más de 300 metros de la red) y considerables para un 2\% (algo menos de 30.000 personas). Probablemente estos enclaves de acceso básico deficitario sean producto de zonas antes despobladas, a las que el sistema nunca llegó. Esto puede encender una señal de alarma en virtud de que algunas zonas que antes se encontraban muy despobladas hoy comienzan a recibir habitantes.

Al desagregar la información anterior por estrato socioeconómico, es posible observar que quienes deben recorrer mayores distancias son los residentes en las zonas con mayor concentración de sectores de bajos ingresos ${ }^{4}$. Así, en los casos en que existe un obstáculo moderado de distancia para acceder a la red (ubicación a más de 300 metros), prácticamente la totalidad de las zonas en dicha situación son de estrato socioeconómico bajo (véase el cuadro 2). De la misma forma, cuando el umbral de distancia asciende a 500 metros, una vez más se aprecia que esa minoría muy reducida, con obstáculos de acceso básico, es casi exclusivamente de estrato bajo.

\section{Cuadro 2}

Montevideo: población por estrato socioeconómico según umbrales de distancia a la red de transporte público

(En porcentajes)

\begin{tabular}{lcccc}
\hline Distancia a la parada & $\begin{array}{c}\text { Alta intensidad de } \\
\text { hogares vulnerables }\end{array}$ & $\begin{array}{c}\text { Intensidad media de } \\
\text { hogares vulnerables }\end{array}$ & $\begin{array}{c}\text { Baja intensidad de } \\
\text { hogares vulnerables }\end{array}$ & Total \\
\hline Más de 300 metros & 16,1 & 3,1 & 1,4 & 8,3 \\
\hline Más de 500 metros & 4,0 & 0,1 & 0,0 & 1,8 \\
\hline Más de 750 metros & 1,1 & 0,0 & 0,0 & 0,5 \\
\hline Distancia media & 204 & 118 & 114 & 162 \\
\hline
\end{tabular}

Fuente: Elaboración propia, sobre la base de información de la Intendencia de Montevideo y el Instituto Nacional de Estadística.

Para avanzar en el análisis de la red más allá de la conectividad, se debe comenzar a considerar variables que especifiquen sus rasgos. Una de las formas de hacerlo es atendiendo a la cantidad de servicios que transitan por la ciudad, medida que da cuenta de la intensidad con que el sistema de transporte recorre la ciudad y la sirve. Por lo tanto, interesará conocer la forma en que se distribuye ese nivel de servicio - o intensidad - a lo largo de la jornada para saber cuáles son los momentos en que se podría inferir que existen mayores dificultades para desplazarse por medio del transporte público.

\footnotetext{
4 El estrato se determina por la composición socioeconómica del segmento censal en el que está ubicada la zona censal. El estrato bajo es aquel en el que reside un $50 \%$ o más de los hogares correspondientes a los quintiles I y II de ingresos. El estrato medio se compone de las zonas con entre un $20 \%$ y un $49 \%$ de hogares en dichos quintiles y las zonas de nivel alto tienen menos del $20 \%$ de los hogares en esa situación. Esta medida, más que sintetizar el estrato socioeconómico de las zonas, refleja la incidencia de población pobre y vulnerable a la pobreza en ese territorio. Así, las zonas consideradas como estrato alto dan cuenta de que en ellas reside una vasta mayoría de hogares cuya vulnerabilidad es baja.
} 
En el gráfico 1 se toma como referencia la cantidad de salidas o largadas en las cabeceras de ambos sentidos para todos los recorridos que componen el sistema. Se registra el conjunto del sistema, sin distinguir direcciones ni recorridos, para saber cuánta es la presencia del transporte en la ciudad, sin determinar aspectos vinculados a la distribución de dicha presencia. Los datos arrojan algunos elementos primarios que tienen que ver con la cantidad de servicio en los distintos regímenes horarios (días hábiles, sábados y domingos y feriados). Es clara la concentración de servicios en horas punta de los días hábiles, cuando la frecuencia prácticamente duplica la de los restantes días y horarios. Una vez más, la estructura de servicios sigue a la mayor demanda.

\section{Gráfico 1}

Montevideo: intensidad de servicios del sistema de transporte público urbano por hora simple de salida y régimen horario, 2010

(En cantidad de salidas)

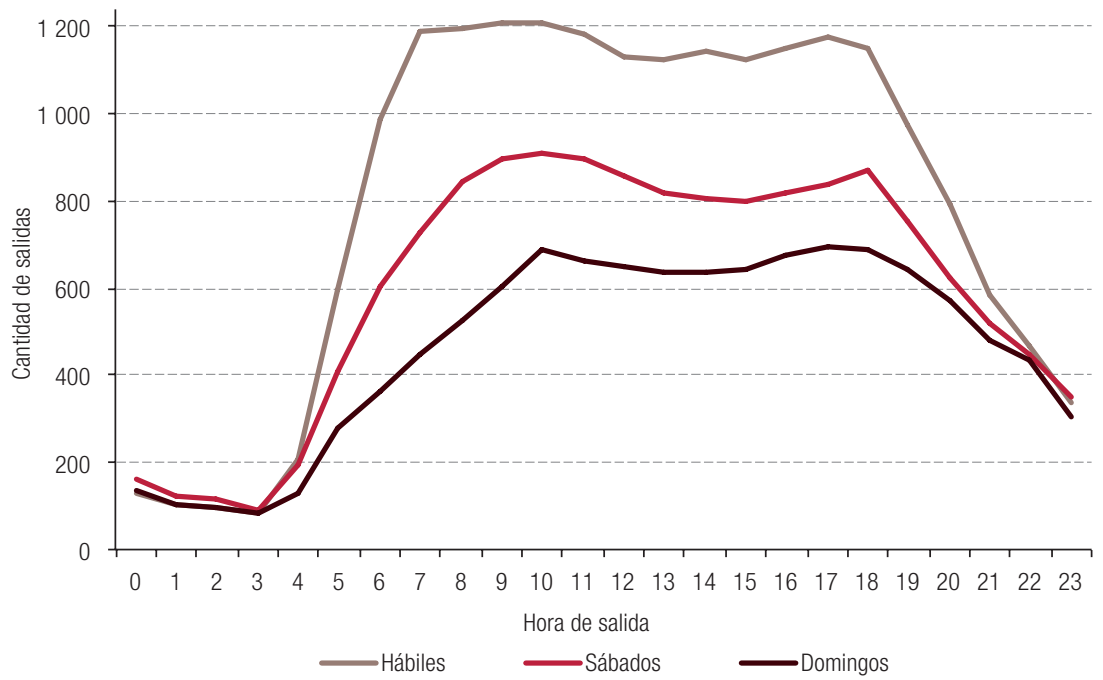

Fuente: Elaboración propia, sobre la base de frecuencias programadas publicadas en la página web de la Intendencia de Montevideo.

\section{Gasto de los hogares en transporte}

Hasta aquí se ha presentado evidencia acerca de la disponibilidad espacial de la red, lo que no garantiza necesariamente su uso. Uno de los elementos que condicionan dicho uso es la capacidad de pago, que es de carácter no espacial y que también define el acceso básico a la red de transporte público. Por tanto, resulta de capital importancia incluir en el análisis el peso monetario que supone el acceso al servicio.

Como afirma Thompson (2002), el transporte urbano representa un costo elevado para los países y las familias. Dicho costo tiene un componente básico constituido por los viajes "obligados" y otro de carácter "opcional" que tiene que ver con la elección de modos de mayor o menor calidad. El costo asumido por las familias aporta pistas sobre la capacidad de los hogares para pagar por el transporte público, así como sobre la forma en que se distribuyen dichos costos entre los estratos sociales y usuarios de distintos modos (Carruthers, Dick y Saurkar, 2005; Falavigna, Nassi y Peixoto, 2011; Serebrisky y otros, 2009). El análisis de la estructura de gastos de los hogares permite inferir cuánta presión monetaria pone la movilidad motorizada sobre las familias de los distintos estratos socioeconómicos. Para ello, por una parte, deberá tenerse en cuenta el porcentaje de los ingresos que es efectivamente utilizado para consumir productos y servicios relacionados al transporte. Por la otra, hay que ver cuáles son los bienes con los que "compiten" los gastos de transporte, es decir, del 
dinero que se gasta en el hogar, cuánto se destina a bienes básicos -y relativamente insustituibles y cuánto se asigna a bienes que, de no ser consumidos, es probable que no impliquen un gran compromiso para el bienestar de los hogares.

Al observar la estructura de gastos de los hogares de Montevideo se destacan los rubros de alimentación, vivienda, salud y transporte y el ítem correspondiente al ahorro, y los dos primeros son los que mayores porcentajes registran a nivel del conjunto de la población (véase el cuadro 3). Al considerar la información discriminada por los ingresos de los hogares, la estructura del gasto se trastoca. En el caso de la alimentación, la distribución por estrato de ingresos es notoria. Entre los hogares del primer quintil este rubro compromete, en promedio, más de la tercera parte de los ingresos, al tiempo que al transitar los quintiles de forma ascendente, su importancia va disminuyendo hasta prácticamente una décima parte de los ingresos corrientes de los hogares del quintil superior. El otro rubro de relevancia -el de la vivienda - presenta una estructura algo más pareja entre los quintiles de ingresos, en tanto el porcentaje dedicado a este ítem solo es algo superior en los tres primeros respecto de los dos restantes. En cuanto a los demás rubros significativos como porción del ingreso de los hogares, el transporte también presenta una distribución con baja asociación al nivel de ingreso y exhibe guarismos algo superiores en los hogares más ricos, tema en el que se ahondará más adelante.

Cuadro 3

Montevideo (promedio simple): gasto porcentual sobre el total de ingreso de los hogares, por rubros de gasto, según quintiles de ingreso, 2006

\begin{tabular}{lrrrrrr}
\hline Rubro de gasto (en porcentajes) & 1 & 2 & 3 & 4 & 5 & Total \\
\hline Vivienda & 28,2 & 28,4 & 27,5 & 24,7 & 24,9 & 26,7 \\
\hline Alimentos y bebidas no alcohólicas & 34,8 & 23,7 & 18,6 & 15,3 & 10,6 & 20,6 \\
\hline Transporte & 7,7 & 7,8 & 7,8 & 8,4 & 8,6 & 8,1 \\
\hline Salud & 3,2 & 7,9 & 10,0 & 10,1 & 8,7 & 8,0 \\
\hline Recreación y cultura & 4,7 & 4,5 & 4,7 & 6,0 & 6,4 & 5,3 \\
\hline Bienes y servicios diversos & 5,8 & 4,3 & 4,0 & 4,0 & 4,1 & 4,5 \\
\hline Comunicación & 4,5 & 4,7 & 4,7 & 4,2 & 3,9 & 4,4 \\
\hline Muebles, artículos para el hogar y su conservación & 4,3 & 3,0 & 3,1 & 3,2 & 4,5 & 3,6 \\
\hline Vestimenta y calzado & 4,5 & 3,1 & 3,2 & 3,2 & 2,9 & 3,4 \\
\hline Restaurantes y servicios de alojamiento & 1,6 & 1,8 & 2,8 & 3,3 & 4,3 & 2,8 \\
\hline Educación & 0,5 & 1,1 & 2,2 & 2,5 & 2,2 & 1,7 \\
\hline Bebidas alcohólicas, tabaco y estupefacientes & 2,3 & 1,8 & 1,7 & 1,3 & 1,0 & 1,6 \\
\hline Gastos no de consumo & 1,0 & 1,2 & 1,4 & 1,7 & 2,6 & 1,6 \\
\hline Ahorro & $-2,5$ & 7,1 & 8,9 & 12,4 & 16,2 & 8,4 \\
\hline
\end{tabular}

Fuente: Elaboración propia, sobre la base de datos de la Encuesta Nacional de Gastos e Ingresos 2006 del Instituto Nacional de Estadística.

En el caso de la salud, la asociación es más perceptible y es directa: los hogares más ricos tienden a gastar una mayor proporción en este rubro que los más pobres. En este caso existe un quiebre a partir del segundo quintil, en el que este gasto asciende, lo que probablemente responda a que en el primer quintil se concentran los usuarios de los servicios de salud estatales gratuitos. En el resto de los quintiles incide la mayor pertenencia al sistema mutual, que implica el pago de cuotas y copagos específicos para obtener determinados servicios. El otro rubro con una esperable y muy alta asociación es el del ahorro de los hogares, esto es, la diferencia entre los ingresos mensuales y los gastos a los que hacen frente. El ahorro adquiere un signo negativo entre los hogares del $20 \%$ de menores ingresos, lo que significa que, en términos proporcionales, los gastos mensuales exceden los 
ingresos. Una vez más, ya a partir del segundo quintil se registra un salto importante en la capacidad de ahorro que se mantiene constante hasta el quintil más rico en el que los niveles de ahorro superan el $16 \%$ de los ingresos.

Las cifras presentadas en el cuadro 3 dan cuenta de una situación marcada por el estrato socioeconómico de los hogares en cuanto a la prioridad respecto de los bienes básicos. Entre los dos quintiles inferiores - en particular en el primero-, es muy notoria la prioridad que se asigna a los bienes alimentarios y a los gastos en vivienda (en este caso, con peso muy similar para todos los estratos). Por supuesto, las razones pueden encontrarse en el propio monto de ingresos sobre los que se dividen los gastos y, en el caso de la alimentación, en el hecho de que en los quintiles inferiores es donde se concentran los hogares más numerosos. Todo esto hace que los bienes básicos ejerzan una enorme presión y hagan más rígido el presupuesto en tanto buena parte de los ingresos será consumida en forma de alimentos. Expresado de otra forma, en los hogares más pobres, el ingreso disponible tras deducir los gastos en comida, alquiler y mantenimiento de la vivienda es muy reducido. Y es en este espacio donde debe acomodarse el gasto en transporte, por lo que, si es alto, competirá con los bienes básicos mencionados.

Como ya se adelantó, más allá de la alimentación y la vivienda, en otros rubros (como salud y educación) se manifiesta la huella de la matriz de protección social. Los hogares más pobres no parecen tener que destinar una enorme parte de sus ingresos a estos rubros (lo que indicaría que los obtienen del Estado), al tiempo que los hogares más ricos sí lo hacen en tanto los consumen desde el mercado. Claro está que en estos casos, la inversión diferencial puede traducirse en diferenciales de calidad; no es novedoso pensar que varias dimensiones (de mayor o menor relevancia) son más cuidadas desde las prestaciones obtenidas en el mercado que en las brindadas gratuitamente desde el Estado.

Al concentrar el análisis en el transporte urbano, es posible observar que para los hogares más pobres, el grueso de lo gastado en transporte urbano corresponde a viajes realizados dentro de la ciudad 5 .

Dentro de los gastos en transporte urbano, la porción más significativa en los primeros tres quintiles se concentra en el transporte público. Esta situación comienza a revertirse en el quintil IV y es prácticamente inversa en el quintil $\bigvee$ (véase el gráfico 2). De la misma forma, es en los quintiles superiores, en particular en el quinto, donde existen porcentajes más importantes de gastos en el subrubro "Otros transporte)". Cuando se hace referencia a los hogares de menores ingresos en relación con el consumo en transporte debe tenerse presente que, en el extremo inferior, prácticamente nueve de cada diez unidades monetarias fueron utilizadas para desplazamientos en la ciudad, al tiempo que casi siete de cada diez se destinaron a pagar los costos del transporte público. Como con otros bienes, se podría especular que los hogares más pudientes consumen servicios de mayor calidad fruto de utilizar el transporte privado, que les garantiza mayor comodidad, velocidad y flexibilidad de destinos y horarios. La diferencia en el caso del transporte urbano es que para estos bienes, los hogares más pobres pagan la misma proporción de sus ingresos que los más ricos.

\footnotetext{
5 A los efectos de la especificación de este rubro, los gastos se clasifican en virtud de los siguientes componentes para cada categoría: a) transporte público: boleto de ómnibus, transporte combinado de pasajeros en distintos medios, transporte interdepartamental (no con fines de esparcimiento) y pasaje de tren; b) transporte privado: accesorios y repuestos de auto, accesorios y repuestos de moto y bicicleta, alquiler de garaje y cochera por mes, servicio de diagnóstico (Computest), reparación de chapa y pintura, estacionamiento (no por mes), gasoil para transporte personal, servicio de gomería, lavado y engrase, licencia de conducir, lubricantes, aceite y cambio de aceite, nafta para transporte personal, neumáticos, cámaras, cubiertas, patente de rodado, reparaciones mecánicas de vehículos, servicio de auxilio de automóvil (cuota mensual), servicio de remolque de vehículo y servicio de transporte escolar; c) otros transportes: alquiler de automóviles sin conductor, alquiler de motos y bicicletas, compra de automóvil o camioneta, compra de bicicleta, compra de moto de menos de 50 cc, empadronamiento de vehículo, fletes, gastos menores de transporte en viajes, lecciones para conducir, servicio de mudanza, pasaje de ómnibus internacional, pasaje de avión o barco, peajes y transporte interdepartamental (con fines de esparcimiento). Dentro del rubro transporte público también se computan los gastos correspondientes al transporte no motorizado (caballos para transporte personal, compra de otros equipos de transporte personal, reparación de bicicletas y vehículos sin motor). Los gastos en transporte público individual (taxis y remises) se cuentan dentro del gasto en transporte privado.
} 


\section{Gráfico 2}

Montevideo: composición del gasto dentro del rubro transporte por subrubro, según quintil de ingresos per cápita

(En porcentajes)

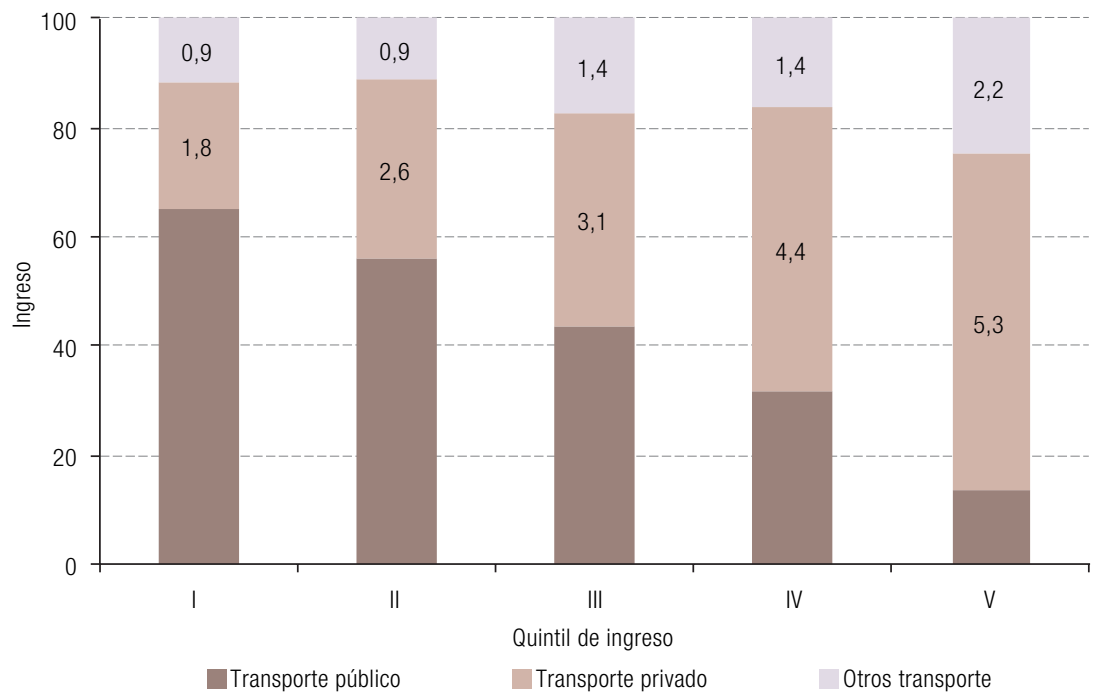

Fuente: Elaboración propia, sobre la base de datos de la Encuesta Nacional de Gastos e Ingresos 2006 del Instituto Nacional de Estadística.

Como ya se mencionó, en los hogares más pobres, la "competencia" del gasto en transporte son los bienes claramente más básicos. En el gráfico 3 se presenta la noción de incidencia competitiva del transporte sobre bienes básicos, que intenta, justamente, capturar algunas de las tensiones a las que se enfrentan los hogares con menores recursos y estimar cuánto amenaza su presupuesto el actual gasto en transporte o un eventual aumento de este gasto ${ }^{6}$. Cuanto mayor sea la presión, más amenazado se verá el consumo de algunos bienes si la necesidad de consumir transporte es inexorable, y, por supuesto, un alto valor de incidencia competitiva muy probablemente va a significar una provisión deficitaria de transporte. Esta podría darse en la forma de suspensión de actividades que requieran erogaciones o bien en la búsqueda de alternativas sin costos monetarios aunque tuviera altos costos personales (por ejemplo, trasladarse grandes distancias a pie).

Los datos son categóricos en cuanto a la distribución regresiva de esta medida, en particular en el caso de los hogares del primer quintil de ingresos donde la proporción de ingresos destinada al rubro transporte urbano constituye un $110 \%$ del ingreso disponible. Esta cifra disminuye a menos de la mitad ya en los hogares del segundo quintil y continúa decreciendo a medida que aumentan los ingresos, lo que determina una situación con amplia holgura para satisfacer las necesidades de transporte y ampliarlas sin comprometer otros consumos. Otro de los rasgos del comportamiento de esta variable es que al considerar tanto el rubro de transporte en su totalidad como el transporte urbano, en el quintil inferior la incidencia competitiva es similar. A medida que se eleva el quintil de ingresos esta diferencia es, en términos proporcionales, mucho mayor.

\footnotetext{
6 Esta medida es el cociente simple del porcentaje de ingresos destinados a transporte sobre la proporción destinada a gastos sustituibles (ocio, esparcimiento y ahorro).
} 
Gráfico 3

Montevideo: medida de incidencia competitiva del rubro transporte urbano sobre bienes básicos, 2006

(En proporción del gasto en transporte sobre el gasto sustituible)

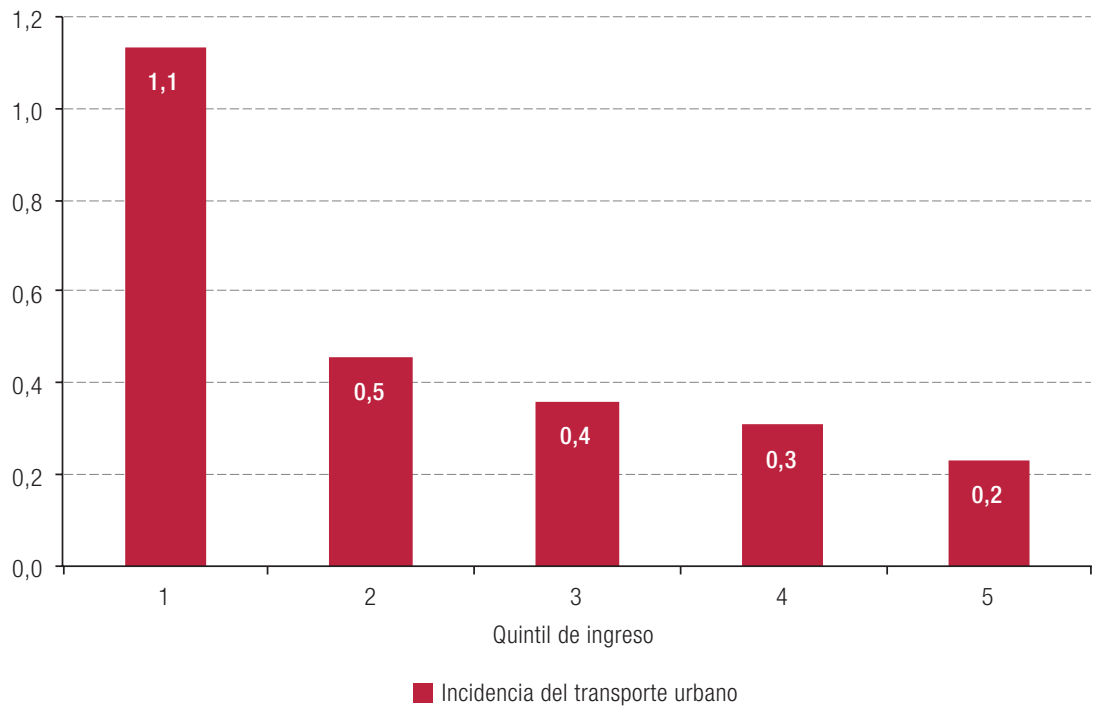

Fuente: Elaboración propia, sobre la base de datos de la Encuesta Nacional de Gastos e Ingresos 2006 del Instituto Nacional de Estadística.

Otro elemento que debe tenerse presente a la hora de analizar los gastos de transporte tiene que ver con la cantidad de personas que viven en el hogar. Esta es una forma distinta de ver el mismo fenómeno estudiado hasta aquí, en tanto la cantidad de personas está asociada con los gastos de alimentación, que conforman una parte importante del conjunto de bienes básicos y es uno de los rubros donde existen mayores diferencias absolutas entre los distintos quintiles, que de cierta forma definen el porcentaje de ingreso disponible. El indicador elegido para integrar la cantidad de personas que viven en los hogares al análisis es la razón del gasto en transporte sobre el ingreso per cápita. De esta manera, es posible estandarizar el gasto en transporte urbano por la cantidad de miembros del hogar. En otras palabras, con este dato se puede dar cuenta del ingreso posterior al gasto en transporte ajustado por la cantidad de personas a las que se tiene que mantener con él. Si bien este dato podría tener que ver con que en los hogares más pobres haya más personas para transportar (en ese caso, a un costo de transporte per cápita - como proporción de los ingresos - mucho más barato que en los hogares más ricos, lo que redundaría en una misma proporción de gastos sobre ingreso total), el efecto de esta mayor demanda es limitado en tanto estos hogares, además de ser más grandes, concentran un mayor número de niños cuyas necesidades de transporte, en general, se satisfacen a través de medios no motorizados ${ }^{7}$.

Al observar la distribución de este indicador por quintiles, se advierte una pauta regresiva, donde el gasto sobre ingreso per cápita en los hogares del primer quintil dobla al del quinto (véase el gráfico 4). En otras palabras, el lugar que el transporte ocupa es mucho más importante entre los hogares pobres cuando se considera la cantidad de personas que se debe atender con el ingreso disponible posterior al gasto en transporte.

\footnotetext{
7 El promedio de personas que viven en los hogares, por quintil de ingresos, es el siguiente: 4,4 personas en el quintil I, 3,4 personas en el quintil II, 2,8 personas en el quintil III, 2,4 personas en el quintil IV y 2,1 personas en el quintil V. En relación con la cantidad de niños y jóvenes de 0 a 14 años, en los hogares del quintil I este grupo etario representa, en promedio, el $41 \%$ de sus miembros, en el quintil II equivale al 26\%, en el quintil III representa el 16\%, en el quintil IV equivale al $12 \%$ y en el quintil $\checkmark$ representa el 10\%. Estos dos indicadores surgen de la Encuesta Continua de Hogares 2007 y corresponden a Montevideo (con quintiles de ingreso per cápita del hogar computados sobre el total del país).
} 


\section{Gráfico 4}

Montevideo: gasto en transporte urbano por quintil de ingreso, 2006

(En porcentajes)

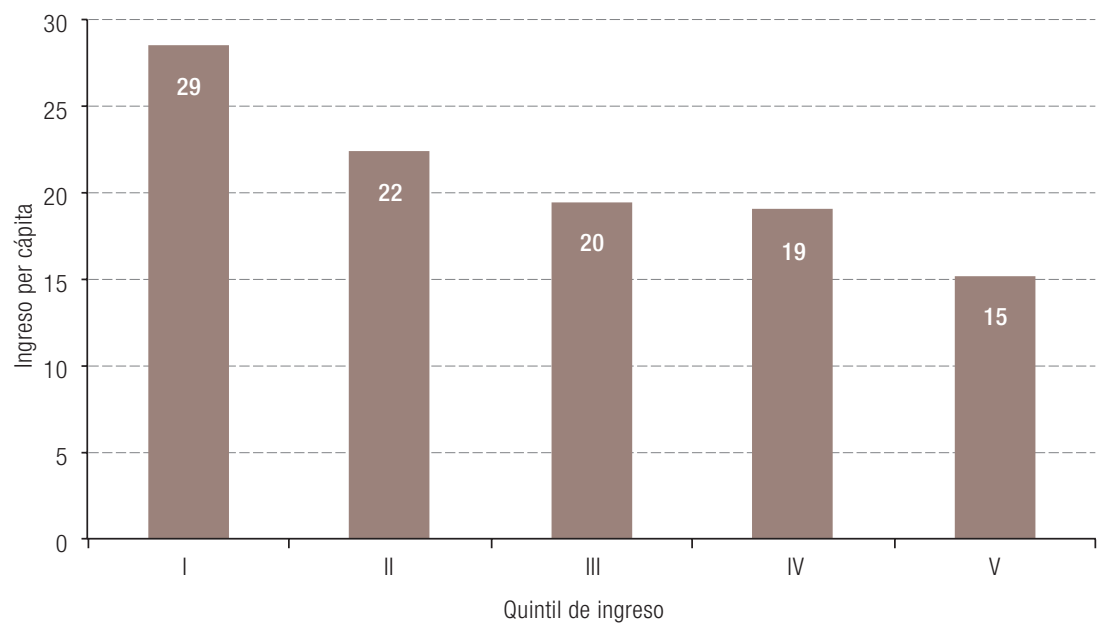

Fuente: Elaboración propia, sobre la base de datos de la Encuesta Nacional de Gastos e Ingresos 2006 del Instituto Nacional de Estadística.

\section{Mecanismos de desmercantilización financiera}

La participación del Estado en la definición de la tarifa es crucial si existe alguna intención de echar a andar mecanismos compensatorios con mayor o menor nivel de focalización. La autoridad puede participar a través de la contribución de fondos públicos para apoyar el funcionamiento del sistema - subsidios - o a partir de regulaciones que impliquen ofertas diferenciadas - estructura tarifariaque favorecen intencionalmente o por los hábitos de uso a sectores específicos.

Como ocurre con otros servicios básicos (energía eléctrica, agua o saneamiento), existen varios puntos de vista acerca de cuál es la mejor estrategia en esta materia. Más allá de diversas posiciones acerca de cuál es la mejor forma de implementar subsidios al transporte público, en todo caso, se reconoce el papel relevante que estos pueden llegar a tener en términos de acceso al transporte. Los sistemas de transporte enfrentarán los mismos dilemas en cuanto a su efectividad, eficacia, conveniencia e impacto. Estos temas se abordan en investigaciones regionales que se focalizan principalmente en el impacto sobre la capacidad de pago del transporte (Carruthers, Dick y Saurkar, 2005; Estupiñán y otros, 2007; Gómez-Lobo, 2007; Gomide, Leite y Rebelo, 2005; Gutiérrez, 2004; Rogat, 2009; Serebrisky y otros, 2009)8.

En Montevideo se podrían identificar tres tipos de mecanismos de desmercantilización financiera. El primero de ellos es de carácter universal y su componente más relevante es el subsidio para incidir a la baja en la tarifa general. Los presuntos beneficiados de estas transferencias de fondos públicos son los usuarios del transporte público en general que, como en otras ciudades latinoamericanas, presentan un perfil en forma de "U invertida". Esto significa que el porcentaje de utilización es muy bajo en los sectores más bajos y asciende entre los sectores medios y medio-bajos para después comenzar a descender en los sectores medio-altos y altos, donde se vuelve al porcentaje mínimo de uso. En el gráfico 5 se da cuenta de este patrón de utilización.

8 Existen, además, varios argumentos económicos que fundamentan la necesidad de subsidios no en razones de interés social, sino en las características del mercado del transporte. Véase una profundización de estos argumentos en Pucher, Markstedt y Hirschman (1983), Serebrisky y otros (2009), y Basso y Silva (2014). 


\section{Gráfico 5}

Montevideo metropolitano: estructura de viajes en transporte público ordenada por percentil de ingresos, 2009

(En porcentajes acumulados)

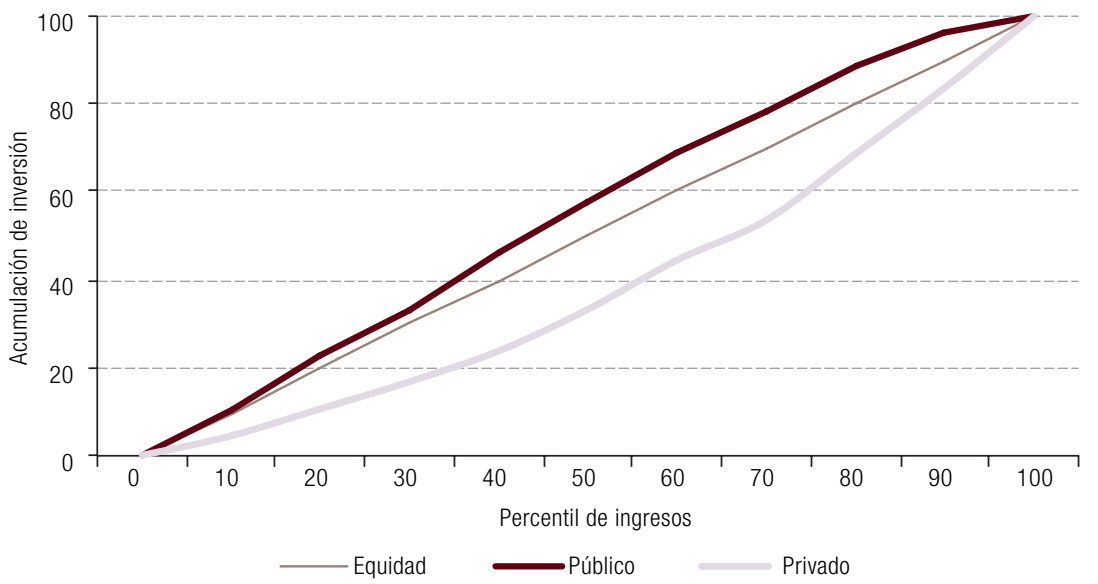

Fuente: Elaboración propia, sobre la base de datos de la Encuesta Origen-Destino (EOD) 2009.

El hecho destacable, de todas formas, es que si el mecanismo de focalización fuera el de las personas que viajan en transporte público, se alcanzaría una distribución con sesgo levemente progresivo (coeficiente de concentración de -0,10). Es así que, por ejemplo, el $40 \%$ de los viajes "más pobres" recibirían cerca del $50 \%$ del subsidio si la asignación subsidio-usuario fuera perfecta. También se pone de manifiesto que dicha progresividad emerge sobre el décimo percentil de ingresos y no antes, donde la línea de viajes de transporte público se apoya sobre la de equidad (cada decil recibiría el $10 \%$ de los subsidios).

Hay que destacar que este dispositivo también incide en los restantes que se enumeran a continuación. Esto se debe a que el subsidio opera sobre la definición de la tarifa básica, que, a su vez, es insumo para el cálculo del resto de las tarifas. Por ejemplo, el boleto bonificado para jubilados equivaldrá a 0,5 tarifas comunes, por lo que, en los hechos, también se beneficia del subsidio general.

El segundo mecanismo es el de beneficios a colectivos específicos. Dentro de esta categoría se encuentran un conjunto de bonificaciones respaldadas por un subsidio municipal y otras que son costeadas de forma cruzada, esto es, por la recaudación del pago del resto de los usuarios. Los del primer tipo incluyen tarifas bonificadas a jubilados y retirados que implican descuentos del $50 \%$ o el $75 \%$, dependiendo de los ingresos jubilatorios. En este caso, abarca únicamente a las personas que reciban alguna prestación jubilatoria o de pensión. También se compone de tarifas diferenciadas para estudiantes de enseñanza media y terciaria, que consisten en un descuento que va del $50 \%$ al 100\%, dependiendo del nivel educativo y la edad del beneficiario.

Las bonificaciones totales que surgen de subsidios cruzados incluyen la gratuidad para estudiantes de enseñanza primaria y para mayores de 70 años y menores de 12 años los domingos. La gratuidad para escolares ha sido una práctica tradicional en el transporte colectivo montevideano, al tiempo que la exoneración por motivos de esparcimiento para niños y mayores fue implementada después de 1990.

Los mecanismos basados en grupos sociales sufren el efecto de autoselección. En el caso de los estudiantes esto es claro: los niños o jóvenes que no asisten al sistema educativo se concentran en los sectores más populares. También es cierto que la incidencia de la caminata en los viajes hacia y desde los centros de estudio, especialmente hasta el primer ciclo de enseñanza secundaria, es muy alta. La bonificación a jubilados y pensionistas puede padecer un efecto similar en relación con ciertos 
requisitos mínimos de formalidad - debe tramitarse un carné en el instituto previsional - de los que se pueden llegar a ver excluidos los sectores más pobres.

Por último, el tercer tipo de mecanismo identificado es el de base territorial y temporal, que surge de la diversificación de la oferta tarifaria a los pasajeros. Con la compra de alguna de estas modalidades, se puede realizar un viaje - o recorrer más tramos de un mismo viaje - por menos dinero que el que correspondería si se adquiriera un boleto o la cantidad de boletos equivalentes a precio regular. Este mecanismo puede interpretarse en dos dimensiones: temporal y territorial. Dentro de la dimensión temporal se cuenta la oferta de dos productos diferenciados de tarifa plana y semiplana por un lapso de tiempo: el boleto "2 horas", que permite utilizar libremente el sistema durante ese lapso por un valor aproximado a 1,4 boletos regulares, y el boleto "1 hora", que permite realizar hasta dos viajes en el transporte colectivo por el valor de la tarifa normal ${ }^{9}$. Por eso, estrictamente hablando, la tarifa regular (el llamado "boleto común") en realidad es una tarifa semiplana de una hora. Esta estructura tarifaria favorece a aquellos que, en primer lugar, usan la tarifa y, en segundo término, más aprovechan sus posibilidades en relación con los viajes realizados. Es así que quienes hacen los dos viajes con un único boleto son financiados por quienes realizan un viaje simple. Algo similar sucede con quienes hacen varios viajes pagando el valor de un boleto de dos horas. Existe un subsidio cruzado entre quienes utilizan esta tarifa y quienes no, al igual que entre los usuarios más intensivos respecto de los que menos la usan ${ }^{10}$.

En la dimensión espacial se destacan los recorridos de carácter zonal o local, cuya bonificación es del $50 \%$ de la tarifa regular. Entre estos recorridos se cuentan los servicios locales, recorridos cortos en extensión, algunos con carácter circular, que recorren sectores no servidos por la red regular o unen zonas lejanas con puntos de intercambio del sistema. También se incluyen algunas porciones de recorridos regulares que, a partir de determinado punto, emiten boletos con tarifa zonal. La última modalidad incorporada es un anillo periférico que fue catalogado como zonal: todos los viajes que se originen y tengan destino dentro de ese anillo se podrán realizar con una tarifa zonal. Este tipo de tarifa es particularmente progresiva en tanto que la mayoría de los servicios locales se ubican en zonas periféricas donde se concentran sectores de bajos recursos. En especial, el boleto zonal del tercer anillo recién mencionado tiene una particular autoselección de carácter progresivo.

En el cuadro 4 se sintetizan los tres mecanismos analizados, así como cada uno de sus componentes ${ }^{11}$.

9 Esta tarifa permite hasta un tercer viaje cuando algunos de los tramos se aborda en determinados puntos del sistema (en general, terminales o intercambiadores).

${ }^{10}$ A nivel de los operadores, existe un esquema de reparto del ingreso por el que se divide en partes iguales por cada tramo utilizado y cada empresa recibe el equivalente a la cantidad de tramos en que la persona utilizó el sistema.

11 Vale destacar que el sistema de transporte presenta otros dispositivos que no son tenidos en cuenta aquí por su carácter marginal en cuanto a la incidencia en la compra de viajes o beneficiarios. Estos son: el boleto institucional, que permite un descuento en la tarifa por compra de un paquete de boletos para empleados de las empresas que realicen un convenio con la Intendencia de Montevideo, y la gratuidad para algunas personas con discapacidad. 
Cuadro 4

Montevideo: dispositivos compensatorios del transporte público

\begin{tabular}{|c|c|c|}
\hline Tipo de mecanismo & Dispositivo & Descripción (beneficios, focalización, financiamiento) \\
\hline De base universal & $\begin{array}{l}\text { Subsidio a la tarifa regular (fideicomiso } \\
\text { del transporte y subsidio a la tarifa) }\end{array}$ & $\begin{array}{l}\text { Estabilización de tarifa y rebaja puntual (aproximadamente un } \\
10 \% \text { de impacto) }{ }^{\text {a }} \text {. Financiamiento desde el gobierno nacional } \\
\text { y departamental, coordinado por este último. Se focaliza en } \\
\text { usuarios del transporte público y tiene una progresividad } \\
\text { moderada. Define la tarifa base para el resto de los dispositivos. }\end{array}$ \\
\hline \multirow{4}{*}{$\begin{array}{l}\text { Por grupo social } \\
\text { (edad y actividad) }\end{array}$} & Estudiantes de enseñanza primaria & Gratuidad. Financiamiento cruzado. \\
\hline & Estudiantes de enseñanza media & $\begin{array}{l}\text { Sistema público: } 50 \text { boletos gratuitos en el ciclo básico de la } \\
\text { enseñanza media (hasta } 18 \text { años) y en el segundo ciclo (hasta } 20 \\
\text { años). Cuando no se cumple el requisito etario y en instituciones } \\
\text { privadas: } 50 \% \text {. Financiamiento nacional de boletos gratuitos } \\
\text { y financiamiento nacional y municipal de tarifa bonificada. } \\
\text { Efecto de autoselección (asistentes al sistema educativo). }\end{array}$ \\
\hline & Estudiantes de enseñanza terciaria & $\begin{array}{l}\text { Solo sistema público: un } 50 \% \text { de bonificación (hasta } 30 \\
\text { años) y un 25\% de bonificación (más de } 30 \text { años). Efecto de } \\
\text { autoselección regresivo. Financiamiento nacional y municipal } \\
\text { (en el caso del 25\%, la bonificación es solo municipal). }\end{array}$ \\
\hline & Boleto de jubilados y pensionistas & $\begin{array}{l}\text { Un } 50 \% \text { y un } 70 \% \text { de bonificación, dependiendo de los } \\
\text { ingresos. Financiamiento municipal. Posible efecto de } \\
\text { exclusión de sectores más vulnerables por requisito formal. }\end{array}$ \\
\hline \multirow[t]{2}{*}{$\begin{array}{l}\text { De base geográfica } \\
\text { y temporal }\end{array}$} & $\begin{array}{l}\text { Oferta local y zonal (recorridos locales, } \\
\text { tramos de recorridos regulares, } \\
\text { viajes dentro del tercer anillo) }\end{array}$ & $\begin{array}{l}\text { Un } 50 \% \text { de la tarifa. Financiamiento cruzado. Gran } \\
\text { concentración en sectores que residen en zonas periféricas. } \\
\text { Autoselección territorial con efecto progresivo. }\end{array}$ \\
\hline & $\begin{array}{l}\text { Oferta de tarifa plana y semiplana } \\
\text { temporal (boletos de } 1 \text { hora y } 2 \text { horas) }\end{array}$ & $\begin{array}{l}\text { Segundo tramo a tarifa regular y viajes ilimitados por } 2 \\
\text { horas con el valor de aproximadamente } 1,4 \text { de viaje a } \\
\text { tarifa regular. Financiamiento cruzado con reparto entre } \\
\text { operadores. Favorece a sectores más alejados del centro } \\
\text { e implica rebaja de tarifas para trasbordos impuestos. }\end{array}$ \\
\hline
\end{tabular}

Fuente: Elaboración propia, sobre la base de información de la Intendencia de Montevideo.

a De acuerdo con consultas realizadas a técnicos de la Intendencia de Montevideo.

\section{Consideraciones finales}

La evidencia indica que la red de transporte público de Montevideo mantiene una cobertura territorial y de personas muy alta. Su comportamiento denota un elevado grado de adaptación a la demanda o, expresado de otra forma, una oferta que, tanto en lo espacial como en lo temporal, se rige por el seguimiento de la demanda real observada. Es así que, en términos territoriales, la red se concentra en los sectores de mayor densidad poblacional y atracción de viajes, en tanto que en los sectores de menor densidad se concentra en forma radial (con ejes relativamente únicos que dejan amplios intersticios territoriales sin red alguna), aunque en el conjunto de ese territorio más amplio que es la periferia suburbana resida casi una tercera parte de la población. Por otra parte, la dimensión temporal presenta un comportamiento idéntico: las franjas y los regímenes horarios de punta - días hábiles en horas diurnas - presentan prácticamente el doble de servicios que los de fuera de punta (fines de semana y horarios nocturnos y fuera de hora punta). Si bien es marginal, se advierte que los últimos procesos de expansión periférica en la ciudad inciden en que las zonas con peor cobertura tengan un perfil prácticamente exclusivo de zonas con alta intensidad de vulnerabilidad.

Dicho esto, la principal debilidad en materia de estratificación se advierte al considerar la capacidad de pago. El gasto de los hogares presenta una distribución regresiva en lo que respecta al acceso al transporte similar a la que se podría encontrar en otras ciudades (Falavigna, Nassi y Peixoto, 2011; PNUD, 2008; Thompson, 2002). En este ítem, compuesto en su mayor parte por el consumo de transporte público, los hogares de los quintiles inferiores erogan una mayor proporción de sus ingresos que los superiores. Asimismo, al considerar la "competencia" con bienes básicos o la cantidad de personas que viven en estos hogares, dichas diferencias son más significativas. 
En este contexto, la estructura de consumo indica que no se debe soslayar el gasto en transporte en tanto tensa la obtención de otros bienes básicos. A su vez, esto incide en la calidad y cantidad de transporte que se consume, ya que se convierte en un rubro pasible de ser recortado o, en un escenario donde sea indispensable, estos recortes podrían dirigirse a bienes básicos.

Si este análisis fuera sobre el sistema de salud, estudiaríamos los llamados "gastos de bolsillo": la cantidad de dinero que los hogares deben desembolsar para acceder a la salud. En este campo, los sectores más favorecidos en general acceden a opciones privadas y de mejor calidad, lo que se refleja en una proporción mayor de gasto en relación con sus ingresos. En el caso del transporte esto no sucede. Si bien los sectores medios y altos también acceden a opciones privadas de mejor calidad -y seguramente realizan una mayor cantidad de desplazamientos a destinos diversos en la ciudad-, en términos proporcionales, usan menos de sus ingresos (o lo mismo) que el resto de los hogares. Esta situación se registra a pesar del esfuerzo de las autoridades -importante en términos regionales - por intervenir la tarifa a la baja y maximizar la capacidad de pago.

Los resultados de la investigación alientan la adopción de un enfoque regional a la hora de analizar la accesibilidad y las políticas de transporte público. Primero, porque la capacidad de pago del transporte es un tema pendiente en la región, lo que apunta al riesgo de precarización de sectores populares, ya sea por no poder movilizarse o por tener que hacerlo en condiciones inadecuadas ante la imposibilidad de pagar el servicio. En segundo término, porque, a diferencia de Montevideo, en varias ciudades latinoamericanas la cobertura básica aún es un problema pendiente (véanse, por ejemplo, CAF, 2009; Bocarejo y Oviedo, 2012; Vasconcellos, 2012). En tercer lugar, porque la cuestión de la calidad es central en toda la región. En este documento no se aborda la situación de la calidad en Montevideo, pero es ineludible para la agenda futura preguntarse a qué nivel de servicio y con qué frecuencia y regularidad habilita el alto nivel de acceso cercano al sistema. Esa pregunta es central a la hora de analizar el grado de desmercantilización.

\section{Bibliografía}

Basso, L. y H.S. Silva (2014), "Efficiency and substitutability of transit subsidies and other urban transport policies", American Economic Journal: Economic Policy, vol. 6, № 4, Nashville, Tennessee, American Economic Association.

Bocarejo, J.P. y D.R. Oviedo (2012), "Transport accessibility and social inequities: a tool for identification of mobility needs and evaluation of transport investments", Journal of Transport Geography, vol. 24, Amsterdam, Elsevier.

CAF (Corporación Andina de Fomento) (2009), Observatorio de Movilidad Urbana para América Latina. Información para mejores políticas y mejores ciudades, Caracas.

Carruthers, R., M. Dick y A. Saurkar (2005), "Affordability of public transport in developing countries", Transport Papers, N ${ }^{\circ}$ 3, Washington, D.C., Banco Mundial.

Cass, N., E. Shove y J. Urry (2005), "Social exclusion, mobility and access", The Sociological Review, vol. 53, $N^{\circ} 3$, Wiley.

Church, A., M. Frost y K. Sullivan (2000), "Transport and social exclusion in London". Transport Policy, vol. 7 , $N^{\circ} 3$, Amsterdam, Elsevier.

Esping-Andersen, G. (1990), The Three Worlds of Welfare Capitalism, Princeton, Princeton University Press.

Estupiñán, N. y otros (2007), "Affordability and subsidies in public urban transport: what do we mean, what can be done?", Policy Research Working Paper, № 4440, Washington, D.C., Banco Mundial.

Falavigna, C., C.D. Nassi y M. Peixoto (2011), "As despesas em transporte e seu impacto no orcamento familiar: um indicador para considerar equidade no planejamento de transportes", documento presentado en el XXIV Congresso de Pesquisa e Ensino em Transportes, Salvador de Bahía.

Flamm, M., C. Jemelin y V. Kaufmann (2008), Travel Behaviour Adaptation Processes during Life Course Transitions. A Methodological and Empirical Study using a Person-based GPS Tracking System, Laboratoire de Sociologie Urbaine (LASUR). 
Gómez-Lobo, A. (2007), "Affordability of public transport: a methodological clarification", Documentos de Trabajo, № 261, Santiago, Universidad de Chile.

Gomide, A., S. Leite y J. Rebelo (2005), "Public transport and urban poverty: a synthetic index of adequate service", Documento de Trabajo, № 33901, Washington, D.C., Banco Mundial.

Gutiérrez, A. (2004), "Concentración empresaria y eficiencia en el autotransporte urbano de Buenos Aires", EURE. Revista Latinoamericana de Estudios Urbano Regionales, vol. 30, № 91, Santiago, Pontificia Universidad Católica de Chile.

Gutiérrez, J. y J.C. García (2005), "Sobreestimaciones del cálculo de distancias en línea recta con respecto al de distancias viarias en el análisis de cobertura de las redes de transporte público", Madrid, Universidad Complutense de Madrid.

Hernández, D. (2012), "Activos y estructuras de oportunidades de movilidad. Una propuesta analítica para el estudio de la accesibilidad por transporte público, el bienestar y la equidad", EURE. Revista Latinoamericana de Estudios Urbano Regionales, vol. 38, № 115, Santiago, Pontificia Universidad Católica de Chile.

Intendencia de Montevideo (2010), Plan Montevideo. Memoria de ordenación, Montevideo.

Kaufmann, V. (2002), Re-thinking Mobility. Contemporary Sociology, Aldershot, Ashgate.

Kaufmann, V., M. Bergman y D. Joye (2004), "Motility: mobility as capital", International Journal of Urban and Regional Research, vol. 28, № 4, Wiley.

Kaztman, R. (2009), "Territorio y empleo: circuitos de realimentación de las desigualdades en Montevideo", documento presentado en el XXVIII Congreso Internacional de la Asociación de Estudios Latinoamericanos, Río de Janeiro.

(1999), Activos y estructuras de oportunidades. Estudios sobre las raíces de la vulnerabilidad social en Uruguay (LC/MVD/R.180/E), Montevideo, Programa de las Naciones Unidas para el Desarrollo (PNUD)/ Comisión Económica para América Latina y el Caribe (CEPAL).

Kaztman, R. y F. Filgueira (2006), "Las normas como bien público y como bien privado: reflexiones en las fronteras del enfoque AVEO", Documentos de Trabajo del IPES - Colección Aportes Conceptuales, Nㅜ 4, Montevideo, Universidad Católica del Uruguay.

Manderscheid, K. (2009), "Unequal mobilities", Mobilities and Inequality, Surrey, Ashgate.

Massot, M.-H. y J.-P. Orfeuil (2005), "La mobilite au quotidien, entre choix individuel et production sociale", Cahiers internationaux de sociologie, $\mathrm{N}^{\circ} 118$.

Miralles-Guasch, C. y A. Cebollada (2003), "Movilidad y transporte. Opciones políticas para la ciudad", Documentos de Trabajo, № 25, Barcelona, Laboratorio de Alternativas.

Moser, C. (1998), "The asset vulnerability framework: reassessing urban poverty reduction strategies", World Development, vol. 26, № 1, Amsterdam, Elsevier.

Ohnmacht, T., H. Maksim y M. Bergman (2009), "Mobilities and inequalities - Making connections", Mobilities and Inequality, Surrey, Ashgate.

PNUD (Programa de las Naciones Unidas para el Desarrollo) (2008), "El futuro de la movilidad en Bogotá. Reflexiones a propósito del Plan Maestro de Movilidad y Estacionamientos", Cuadernos del Informe de Desarrollo Humano para Bogotá, № 1, Bogotá.

Pucher, J., A. Markstedt e I. Hirschman (1983), "Impacts of subsidies on the costs of urban public transport", Journal of Transport Economics and Policy, vol. 17, № 2.

Rogat, J. (2009), Regulación y planificación de buses en América Latina. Resumen orientado a tomadores de decisiones, Centro PNUMA Risø.

Serebrisky, T. y otros (2009), "Affordability and subsidies in public urban transport: what do we mean, what can be done?", Transport Reviews, vol. 29, № 6, Taylor \& Francis.

Thompson, I. (2002), "Impacto de las tendencias sociales, económicas y tecnológicas sobre el transporte público: una investigación preliminar en ciudades de América Latina", serie Recursos Naturales e Infraestructura, № 41 (LC/L.1717-P), Santiago, Comisión Económica para América Latina y el Caribe (CEPAL).

Titheridge, H. (2006), "Social exclusion and transport policy", Londres, University College, inédito.

Urry, J. (2007), Mobilities, Cambridge, Polity Press.

Urse, G. y G. Márquez (2009), "Un modelo de gestión alternativo en el transporte urbano: el caso de Montevideo", documento presentado en el XV Congreso Latinoamericano de Transporte Público Urbano (CLATPU), Buenos Aires.

Vasconcellos, E.A. (2012), Transporte urbano y movilidad en los países en desarrollo: reflexiones y propuestas, São Paulo, Instituto Movimiento.

Wenglenski, S. y J.-P. Orfeuil (2006), "The differences in the accesibility to the job market according to the social status and the place of residence in the Paris area", inédito. 